\title{
Post-Launch Calibration and Testing of Space Weather Instruments on GOES-R Satellite
}

\author{
Sivakumara S.K. Tadikonda ${ }^{\mathrm{a} *}$, Cynthia S. Merrow ${ }^{\mathrm{b}}$, Jeffrey A. Kronenwetter ${ }^{\mathrm{c}}$, Gustave J. \\ Comeyne $^{\mathrm{d}}$, Daniel G. Flanagan ${ }^{\mathrm{d}}$, Monica Todirita ${ }^{\mathrm{d}}$ \\ ${ }^{a}$ Constellation Software Engineering Corp., 4640 Forbes Blvd, Suite 201, Lanham, MD 20706;

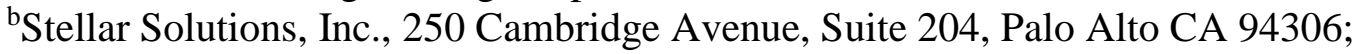 \\ ${ }^{c}$ Chesapeake Aerospace LLC, P.O. Box 436, Grasonville, MD 21638; \\ ${ }^{\mathrm{d}}$ National Oceanic and Atmospheric Administration, NASA/GSFC, Code 417.0, \\ 8800 Greenbelt Rd, Greenbelt, MD 20771
}

\begin{abstract}
The Geostationary Operational Environmental Satellite - R (GOES-R) is the first of a series of satellites to be launched, with the first launch scheduled for October 2016. The three instruments - Solar UltraViolet Imager (SUVI), Extreme ultraviolet and X-ray Irradiance Sensor (EXIS), and Space Environment In-Situ Suite (SEISS) provide the data needed as inputs for the product updates National Oceanic and Atmospheric Administration (NOAA) provides to the public. SUVI is a full-disk extreme ultraviolet imager enabling Active Region characterization, filament eruption, and flare detection. EXIS provides inputs to solar backgrounds/events impacting climate models. SEISS provides particle measurements over a wide energy-and-flux range that varies by several orders of magnitude and these data enable updates to spacecraft charge models for electrostatic discharge. EXIS and SEISS have been tested and calibrated end-toend in ground test facilities around the United States. Due to the complexity of the SUVI design, data from component tests were used in a model to predict on-orbit performance. The ground tests and model updates provided inputs for designing the on-orbit calibration tests. A series of such tests have been planned for the Post-Launch Testing (PLT) of each of these instruments, and specific parameters have been identified that will be updated in the Ground Processing Algorithms, on-orbit parameter tables, or both. Some of SUVI and EXIS calibrations require slewing them off the Sun, while no such maneuvers are needed for SEISS. After a six-month PLT period the GOES-R is expected to be operational. The calibration details are presented in this paper.
\end{abstract}

Keywords: GOES, Space Weather, On-orbit Calibration, EXIS, SEISS, SUVI

\section{INTRODUCTION}

The GOES-R satellite (Figure 1) will be located at 75 deg or 137 deg West longitude for operations at the geostationary locations in the earth's orbit. This is the first of the four satellites in the series that is the next generation to the GOES-13, 14, and 15 series currently in orbit, operated by the National Oceanic and Atmospheric Administration (NOAA). The GOES-R series is being procured by the National Aeronautics and Space Administration (NASA). Lockheed Martin Space Systems Company provides the spacecraft bus and integrates Government-furnished instruments. After launch, spacecraft on-orbit checkout, and instrument calibrations, the satellites will be handed over to NOAA for operations. The first satellite in the GOES-R series is scheduled to be launched in October, 2016.

The GOES-R represents a technological leap forward in satellite meteorology capabilities for earth and space weather prediction $^{1}$, and is required to be fully operational for ten years on-orbit, in addition to a five-year on-orbit storage period. Each satellite consists of an Advanced Baseline Imager (ABI), a Geostationary Lightning Mapper (GLM), a Solar UltraViolet Imager (SUVI), an Extreme ultraviolet and X-ray Irradiance Sensor (EXIS), Space Environment InSitu Suite (SEISS), and a Magnetometer. The ABI and the GLM are nadir-pointed instruments, the EXIS and SUVI are Sun-pointed instruments, the SEISS is spacecraft-body mounted, and a pair of magnetometers are located at the end of 
the magnetometer boom, separated so as to enable a gradiometer approach. Of the Space Weather instruments, EXIS (Figure 2), SUVI (Figure 3), and SEISS (Figure 4), EXIS is an irradiance measuring instrument, SUVI is an imaging instrument, and the SEISS consists of 5 particle count measuring sensors. All of the SEISS sensors are located on the spacecraft bus, complying with the requirements on look-directions. The SUVI and the EXIS are Sun-pointing instruments, and the spacecraft locates them on the Sun-Pointing Platform (SPP) which is gimballed with respect to the solar array. The solar array provides the East-West $(\mathrm{E}-\mathrm{W})$ pointing to the Sun while the gimballed SPP provides the North-South (N-S) pointing. The spacecraft Guidance, Navigation, and Control (GNC) System provides the Sunpointing control using the Solar Array Drive Assembly (SADA) and the Sun Elevation Gimbal Assembly (SEGA) for the E-W and N-S directions, respectively.

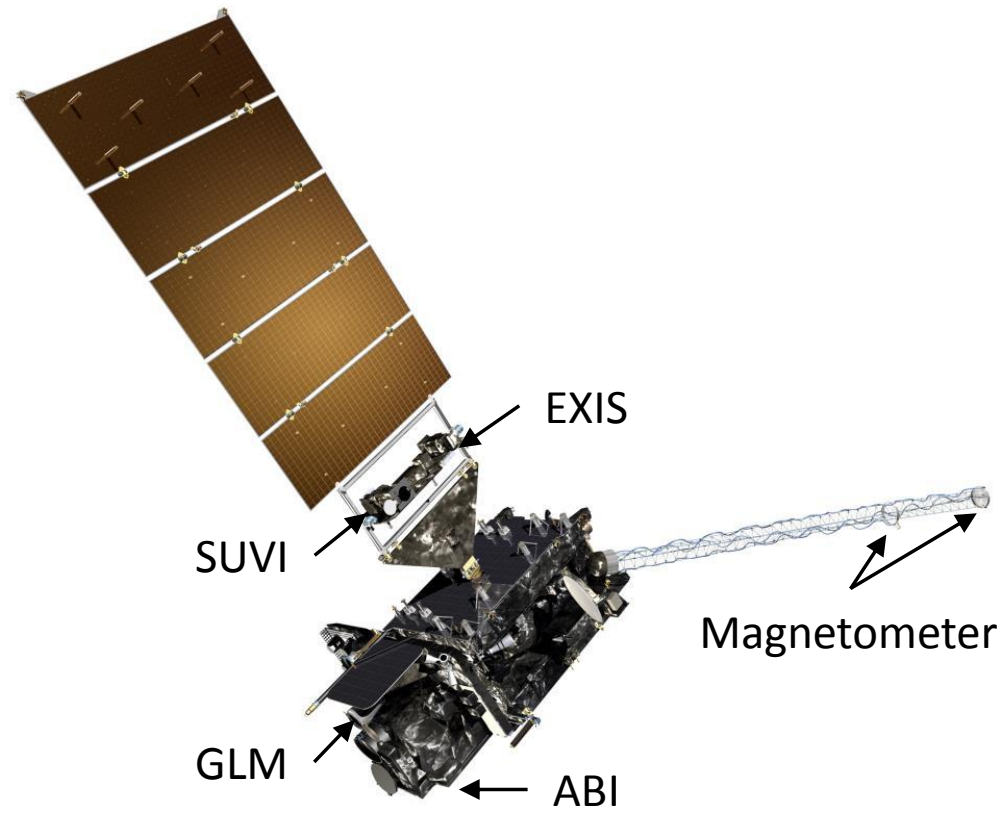

Figure 1: GOES-R spacecraft (Courtesy: Lockheed Martin Space Systems Company)

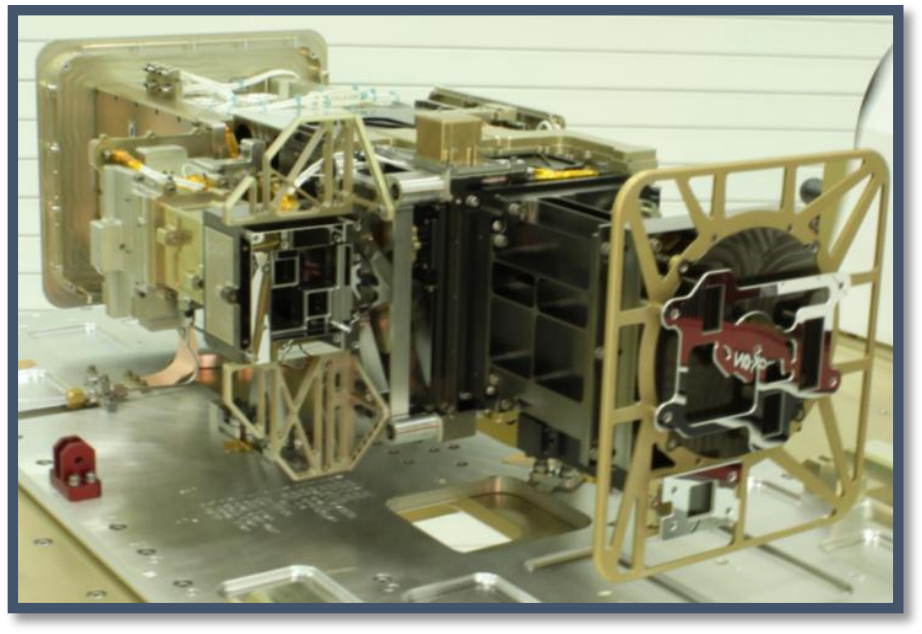

Figure 2: EXIS: Extreme ultraviolet (right) and X-ray (left) Irradiance Sensor (Courtesy: Laboratory for Atmospheric and Space Physics, University of Colorado, Boulder) 
The SEISS consists of: a Magnetospheric Particle Sensor - Low Energy (MPS-Lo) and - High Energy (MPS-Hi), a pair of Solar and Galactic Particle Sensor (SGPS) units, and an Energetic Heavy Ion Sensor (EHIS). The MPS-Lo, MPS-Hi, and EHIS units have their apertures pointed to the zenith. The two SGPS sensor units (SUs) point, one each, to the $+X$ (the direction of the orbital velocity vector) and $-\mathrm{X}$ directions of the spacecraft body axes. With the exception of the $+\mathrm{X}$ SGPS sensor, the remaining SUs are mounted to the spacecraft-provided SEISS cabinet.

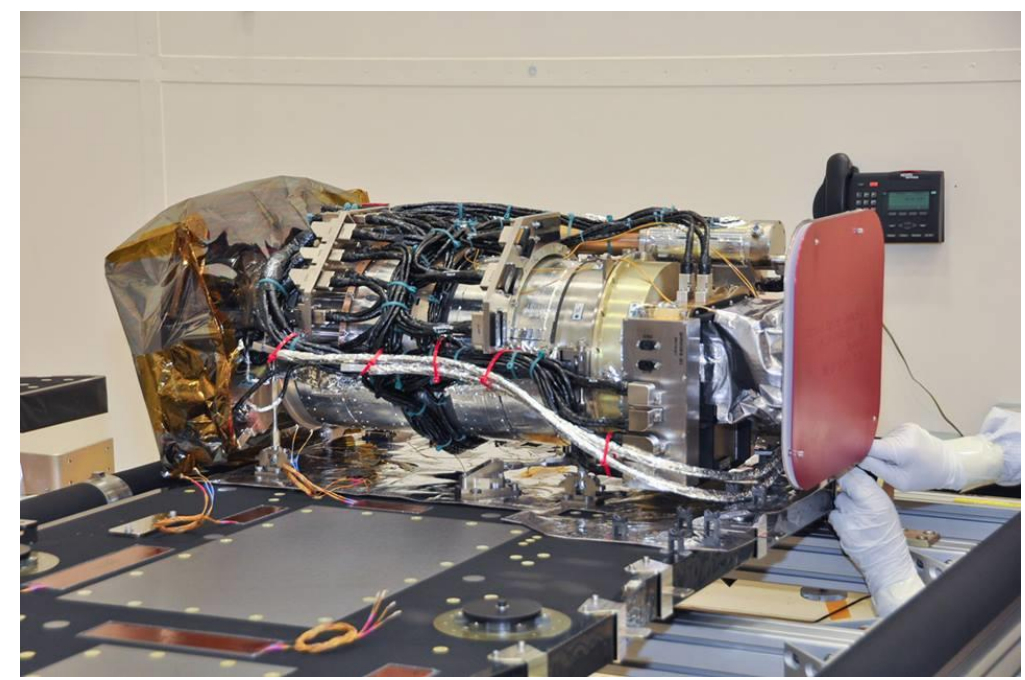

Figure 3: SUVI Telescope System on the Sun-Pointing Platform (Courtesy: Lockheed Martin Space Systems Company)

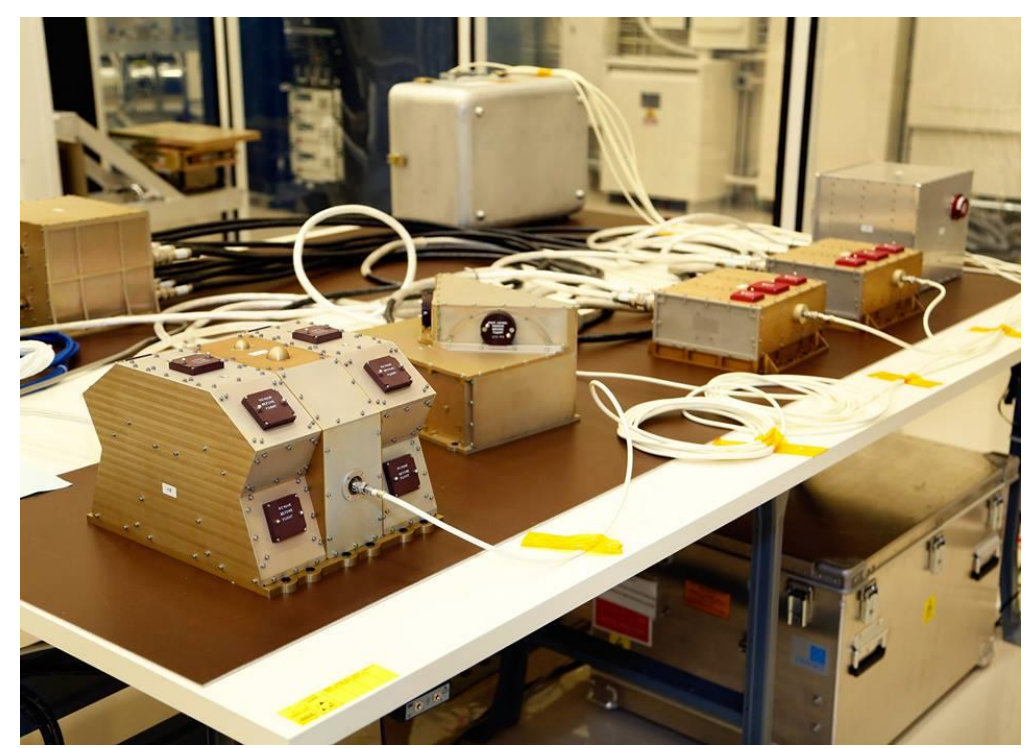

Figure 4: SEISS Instruments on the bench, from left to right: MPS-Hi, MPS-Lo, SGPS-1 \& -2, and EHIS; DPU (back row, left) (Courtesy: Assurance Technology Corporation)

Each of these instruments provides data to the Ground System (GS) via the spacecraft communication system. The GS processes the instrument data, spacecraft telemetry data, orbit determination data and other required information to autonomously generate calibrated and navigated products for the NOAA users. In the GOES-R parlance, these are called Level-1b (L1b) products. 
A prerequisite for on-orbit calibration is ground calibration, complemented by detailed analyses, to provide confidence that instrument performance is compliant with requirements. The on-orbit calibrations then serve to provide updates to parameters used in instrument performance models and to validate the pre-launch predicted performance. The on-orbit calibrations first enable the assessment of the impact of launch. Over the course of the ensuing year, with periodic calibrations, the effect of seasonal variations can be catalogued, and performance parameters can be updated as needed. The GOES-R Program mandated that ground calibrations be performed with National Institute of Standards and Technology (NIST)-traceable sources.

Instrument descriptions are presented next. The ground calibration testing is detailed in Section 3. Brief descriptions of the series of tests planned for the Space Weather instruments after the spacecraft arrival in the orbital slot are provided in Section 4.

\section{INSTRUMENT DESCRIPTIONS}

\subsection{Extreme ultraviolet and X-ray Irradiance Sensor (EXIS)}

EXIS consists of an X-Ray Sensor (XRS) ${ }^{2}$, an Extreme UltraViolet Sensor (EUVS) ${ }^{3}$, and an electronics unit all contained in a single, mechanically integrated package (Figure 2). The purpose of the XRS is to monitor solar flares in order to help predict proton events that can disrupt communications and degrade navigational accuracy. The purpose of the EUVS is to monitor solar variations that directly affect satellite drag / tracking and ionospheric changes, which impact communication and navigation operations.

The XRS consists of three photodiode-based photometer channels, two active (A and B) and one inactive. Channel A covers $0.05-0.4 \mathrm{~nm}$ and channel B covers $0.1-0.8 \mathrm{~nm}$. The "dark" diode channel allow background subtraction. All active channels view the sun through two Beryllium filters. Each active XRS channel consists of a low-sensitivity and a highsensitivity detector whose responses overlap in order to span the required total dynamic range. The low-sensitivity detectors are quadrant photodiodes. They view the sun through a small aperture, and enable the extraction of solar flare position information.

The EUVS consists of three grating spectrographs that will measure different wavelength ranges. The three channels, denoted A, B and C, give coverage in the bands of $16-37 \mathrm{~nm}$ (0.6nm resolution), $115-135 \mathrm{~nm}$ (0.6nm resolution) and 275$285 \mathrm{~nm}(0.1 \mathrm{~nm}$ resolution). From these, the Ground Process Algorithm (GPA) constructs the full solar spectrum for the 5 - $127 \mathrm{~nm}$ wavelength range. Post-dispersion photon detection is done via custom arrays of discrete silicon photodiodes for the A and B channels, and a linear 512-element photodiode array for the $\mathrm{C}$ channel $^{4}$.

\subsection{Solar UltraViolet Imager}

The SUVI instrument leverages the successful design of the Atmospheric Imaging Assembly ${ }^{5}$ flown on the Solar Dynamics Observatory and consists of two units: a SUVI Telescope System (STS) (Figure 3) and a SUVI Electronics Box (SEB) that will be located next to the STS shown in Figure 3, where the mount pattern is clearly visible. The STS consists of a Cassegrain Telescope with normal-incidence, multilayer-coated optics, a Guide Telescope (GT), CCD Detectors, and a Camera Electronics Box (CEB) attached externally to the telescope structure. The STS and the SEB, interconnected by SUVI-provided harnesses, are integrated to the SPP by the spacecraft contractor. SEB provides the power and data interfaces to the spacecraft. The GT provides very accurate Sun position measurement and the spacecraft uses this data for controlling the SPP so that the GT is pointed at the Sun within the accuracy required by SUVI.

SUVI images the Sun in six Extreme UltraViolet (EUV) wavelengths in the $9.4-30.4 \mathrm{~nm} \mathrm{range}^{7}$, with a 10 second cadence, and with a 4 minute refresh for the complete spectrum and the required dynamic range. The primary and secondary mirrors are coated in six equal sectors to provide the respective bandpasses ${ }^{7}$. The fixed entrance filters and the filters on two filter wheels in front of the imaging plane enable both visual light rejection and specific bandpass. The telemetry is then processed by the GPA, applying various corrections such as pointing error, dark current subtraction, bad pixel elimination, and flat-field correction to provide a Sun-centered $40 \operatorname{arcmin}$ x $40 \operatorname{arcmin}$ image. 


\subsection{Space Environmental In-situ Sensor Suite}

The SEISS consists of five SUs listed in Section 1 and a Data Processing Unit (DPU) ${ }^{6}$. The DPU, providing the power and data interfaces to the spacecraft, is located inside the spacecraft bus, while the SUs are mounted externally to the spacecraft bus. The SEISS-provided harnesses are routed through the spacecraft to connect the DPU to the SUs.

The MPS-Lo SU is an Electrostatic Analyzer (ESA) of a triquadrisphere design, and measures $30 \mathrm{eV}$ to $30 \mathrm{keV}$ electrons and ions in 15 bands. The ESA design and a proprietary anti-reflective coating ensure that only particles of the appropriate energy range reach the micro-channel plate (MCP) assemblies ${ }^{6}$.

The MPS-Hi SU measures $50 \mathrm{keV}$ to $4 \mathrm{MeV}$ electrons and protons between $80 \mathrm{keV}$ and $12 \mathrm{MeV}$ in 11 bands. The Fieldof-View (FOV) is achieved using five telescopes for each of the electrons and protons species ${ }^{6}$. The five electron and five proton telescopes are divided between the two banks with two of one species and three of the other in each bank, providing a measure of graceful degradation should a bank fail.

Both MPS-Lo and MPS-Hi measure particles over a FOV that is symmetrical 30 degrees in the orbital plane, and 180 degrees in the $\mathrm{YZ}$ plane, both centered on the zenith axis ${ }^{6}$.

The SGPS measures protons over an energy range of $1-500 \mathrm{MeV}$ in 10 bands with an integral channel for particles greater than $500 \mathrm{MeV}$. The SGPS also measures alpha particle fluxes in the range of $4-500 \mathrm{MeV}^{6}$. As mentioned earlier, there are two SGPS SUs. Each SGPS SU has three telescopes, two with a FOV of $60^{\circ}$ and the third with an FOV of $90^{\circ}$, all centered about the $+/$-X axis. The $-X$ facing SGPS unit is mechanically integrated with the SEISS cabinet while the second unit is located on the $+\mathrm{X}$ face of the spacecraft bus.

The EHIS SU measures protons and heavy ions in the 10 to $200 \mathrm{MeV} /$ nuc energy range for the element groups $\mathrm{H}$, He, $\mathrm{CNO}, \mathrm{Ne}-\mathrm{S}, \mathrm{Fe}(\mathrm{Z}=17-28)$ in five approximately logarithmic energy bands ${ }^{6}$. Its unique design identifies each specific element and outputs the number of ions by element and energy range using the Angle Determining Inclined Sensor (ADIS) system. Accurate incident particle angle information enables the individual elemental separation. The EHIS telescope has a $30^{\circ}$ conical FOV centered about the minus $\mathrm{Z}$ direction ${ }^{6}$.

\section{GROUND CALIBRATIONS}

\subsection{EXIS}

Laboratory for Atmospheric and Space Physics (LASP) employed several sources such as cathode ray lamps, Deuterium lamps, Mercury pen-ray lamps, platinum lamps, tungsten filament lamps, lasers, a Manson Source, with a variety of targets, and a radioactive Fe55 source for preliminary checking as part of the instrument build. Instrument calibration occurred at the Synchrotron Ultraviolet Radiation Facility (SURF) at the NIST campus in Gaithersburg, Maryland. Each of the EXIS instrument Flight Models (FMs) made calibration trips to SURF at NIST for beam testing, both pre- and post-environmental testing, to ensure that the environmental testing did not adversely affect instrument performance, and the results affirmed it. This provided the confidence that the effects of the actual launch and subsequent on-orbit thermal variations have been adequately accounted for. There's an overall uncertainty budget for the XRS and EUVS performance, and these calibrations ensured that responsivity, and values for parameters such as gain and linearity, and dark current stayed within their allocations.

\subsection{SUVI}

SUVI's various components received ground calibrations and the image performance and optical throughput predictions were made using a validated model with data from component calibrations as inputs. Surface roughness measurements were made for the bare primary and the secondary mirrors fabricated by SSG-Tinsley using full aperture interferometry for the low spatial frequencies, a Phase Measuring Microscope (PMM) for the mid spatial frequencies, and an Atomic Force Microscope (AFM) for the high spatial frequencies, to characterize the surface errors ${ }^{7}$. The reflectance of the multi-layer coated primary and secondary mirrors was measured using the Advanced Light Source (ALS) at Lawrence Berkeley National Laboratory (LBNL) $)^{8}$. The CCD calibration was performed in house. Test coupons were used for the entrance and analysis filters supplied by the vendor Luxel for both out-of-band and in-band transmissivity 
characterization. A calibration campaign at the instrument level was not undertaken due to: 1) the fact that a uniformly collimated large aperture beam is needed, and 2) such testing would have required the installation of a non-flight spacer (which would have to be removed post-calibration) between the metering tube and focal plane assemblies to account for the finite distance of the source; such an undertaking, after all the environmental testing was completed, was not considered worth taking the risk due to the fact that the Atmospheric Imaging Assembly (AIA), the precursor to SUVI, on the Solar Dynamics Observatory (SDO) was not calibrated at the instrument-level and the 4 AIA telescopes on SDO continue to perform well as of this writing, thus providing confidence in the SUVI approach. The SUVI optical performance was predicted using a validated empirical model ${ }^{8}$.

\subsection{SEISS}

The wide ranges for the energy, flux, and the type of particles being counted by the SEISS SUs necessitated trips to various facilities. The low-energy and high-flux particle measuring MPS-Lo was characterized at the Calibration Facility at the Air Force Research Laboratory (AFRL) at Kirtland Air Force Base (AFB) in New Mexico. Calibrations for the next-level of particles in the energy-flux curve were performed at NASA Goddard Space Flight Center (GSFC) Radiation Effects Laboratory Accelerators (Van de Graaff \& Cockroft-Walton) Facility and at the Massachusetts Institute of Technology (MIT) Van de Graaff facility, and at the Northeast Proton Therapy Center (NPTC) Cyclotron Facility at the Massachusetts General Hospital (MGH). The SGPS calibration was conducted at the MGH, at the University of California at Davis cyclotron, and at the Brookhaven National Laboratory (BNL). The EHIS calibration, due to the sensor's high energies required specialized accelerator equipment. The MGH facility was used for the proton calibration; and for the heavy ions, the Coupled Cyclotron Facility (CCF) at Michigan State University's (MSU)

National Superconducting Cyclotron Laboratory (NSCL). Since it was not possible to calibrate at every energy level, modeling using GEANT, FLUKA, and SIMION was employed ${ }^{6}$ for interpolation and extrapolation so that the performance compliance can be demonstrated over the entire range specified in the requirements document.

\section{ON-ORBIT CALIBRATIONS}

After instrument-specific outgassing time has elapsed on-orbit, the instrument performance characterization commences. The instrument vendors first perform check-outs of electronics and mechanisms. The focus of the activities listed below is on science characterizations which have also been recommended for periodic execution after the satellite is commissioned for normal operations; this is at the successful conclusion of the PLT phase.

\subsection{EXIS}

\subsubsection{EUVS Stim Lamps and Gains}

With the door closed and with the illumination from an internal LED lamp, data will be collected, to enable trending of the detector gains. The GPA parameters may be updated if the changes exceed thresholds.

\subsubsection{XRS, SPS, and EUVS A/B Darks}

The SPP will be slewed off the Sun by more than 15 degrees to guarantee dark observations. The collected data during the PLT will provide the baseline and this activity is recommended to be performed on a quarterly basis.

\subsubsection{EUVS-A/B Wavelength Scale \& Line Masks}

The diode location of solar emission lines for EUVS-A (25.6, 28.4, and $30.4 \mathrm{~nm})$, EUVS-B (117.5, 121.6, 133.5, and $140.5 \mathrm{~nm}$ ), and the $\mathrm{Mg}$ II core lines for EUVS-C will be determined. For the primary EUVS-C channel, the dark, red wing, and blue wing masks will be determined. From the ensuing analysis results with these data as inputs, the wavelength tables and diode masks used in the GPA for integrating each line will be updated. This is a one-time only calibration. 


\subsubsection{EUVS-C Integration Time Optimization}

The EUVS-C integration period will be set to successively higher values and science data will be obtained for each. Once the optimal period is determined using offline analyses, all RAM and EEPROM stored command tables that use this value are updated. This is a one-time only calibration.

\subsubsection{FOV mapping}

The SPP will be used in a step-and-stare fashion in a $+/-15$ arcmin box around the Sun center at a few randomly selected points that are at least 2 arcmin apart. The FOV map will be developed for each of the channels.

\subsubsection{EUVS Filter Characterization}

The transmissivity of the 24 EUVS filters will be established here. For the positions where EUVS-B and EUVS-C primary channel are considered open, the objective is to collect data confirming that there are no sources of Sun glint or light scatter.

\subsubsection{XRS and EUVS Signal-to-Noise}

XRS and EUVS science data will be collected over several days and a signal-to-noise ratio will be computed for each channel of the XRS and EUVS. The goal is to obtain sufficiently different solar activity levels to allow the extrapolation down to the threshold irradiance levels for each channel. For the XRS, if one long duration flare event occurs, then that would provide sufficient data to perform the extrapolation down to threshold irradiance levels. For the EUVS, solar rotation maximum and minimum are desired. Solar flares would not negatively impact the data used for the EUVS analysis.

\subsubsection{Cruciform Scan slew}

The SPP will be slewed +/-4 degrees of the Sun center in each of the E-W and N-S directions to determine EXIS channel Optical centers.

\subsubsection{XRS Inter-channel Comparison}

Comparisons between similar channels on XRS while pointed at the sun will be conducted. Any relative changes in gain between the channels since ground calibration will produce different values of the solar variability between the channels and these will be catalogued.

\subsection{SUVI}

\subsubsection{CCD Dark Current Characterization}

The CCD dark images are obtained for various temperatures and exposure durations are catalogued. During the normal operations, appropriate images will be selected from the database and subtracted by the Ground Process Algorithm. Although the CCD is planned to be operated at a specific temperature, radiation degradation during the ten-year mission could necessitate raising the operating temperature and the data collected during the PLT will facilitate a table look-up approach for making the required updates.

\subsubsection{Shutter Light Leakage}

With the shutter closed, dark images will be collected for both when the SUVI is facing the Sun and is pointed off the Sun. The difference will enable an assessment of the shutter leakage. This data will be used in the corrections implemented in the GPA.

\subsubsection{Off-Band Signal Characterization}

Each of the entrance and analysis filters provides $10^{-5}$ attenuation for visible light. However, these filters may develop pin-holes during launch. Such pin-holes will significantly increase the amount of off-band signal. Each of the analysis 
filter wheels provides for an open position to enable the pin-hole assessment for the individual filters. Solar images will be collected with specific filter combinations and the ensuing analysis will enable the determination of off-band signal strength.

\subsubsection{Flat-Field Calibration}

The SPP will be pointed to multiple locations on the solar disk and will enable dwell at each location as SUVI collects images. Data to enable the assessment of the flat-field characteristics for each wavelength will be collected. The analysis will be conducted off-line. Two methods - the Kuhn-Lin-Loranz and Boustrophedon - are planned to be employed and their performance will be compared. Only the method that was demonstrated to be superior will be employed for the ensuing periodic characterizations. An outcome of the analysis is the bad pixel map. The charge transfer efficiency will also be determined.

\subsubsection{Focus Check}

The focus mechanism enables an on-orbit adjustment. Although the focus is set prior to launch, true collimated beam is available only on-orbit; the solar images will be collected at various focus settings and the optimum focus will be set after analyzing that data.

\subsubsection{Guide Telescope Calibration}

Owing to the tight SUVI pointing requirements, $+/-1.5$ arcmin of the Sun center, and the fact that spacecraft uses the GT for pointing the SPP to the Sun, the GT calibration has an added significance. Over the linear FOV of the GT, the SPP pointing is offset, both in the E-W and N-S directions. The image will be collected in the CCD, the Sun center will be determined in the image and will be compared with the GT provided data.

\subsubsection{Cross calibration with EXIS}

For the two wavelengths $28.4 \mathrm{~nm}$ and $30.4 \mathrm{~nm}$ for which the EUVS (of EXIS) daily averages of irradiance observations are available, SUVI computes daily irradiances so that CCD contamination accrual can be monitored by comparing with the EUVS data. When it is determined that non-volatile residue contamination has reached a threshold, the CCD temperature is planned to be raised to $55 \mathrm{deg} \mathrm{C}$ to bake it off.

\subsection{SEISS}

\subsubsection{In-Flight Calibrations (IFCs)}

Each of the SEISS SUs is capable of performing an internal calibration via autonomous test sequences for the purpose of determining detector threshold characteristics as a state of health check for telescopes. Post-launch IFCs establish the on-orbit performance baseline for mission life trending. For the MPS-Hi, SGPS, and EHIS SUs, the IFC sequences are determined by IFC Tables stored in on-board memory. The data from the initial IFCs executed post-launch will be compared to pre-launch data and if an update to the on-board IFC sequence is warranted to adjust for the on-orbit environment, then the table can be re-configured on the ground and re-uploaded to update IFC sequence. The MPS-Lo IFC sequence is part of the Field Programmable Gate Array (FPGA) and cannot be updated on-orbit.

\subsubsection{EHIS On-Orbit Calibration}

Due to the fact that it was not possible to fully calibrate the EHIS SU during ground calibration, i.e. particles in the high energy ranges are not available at the ground calibration facility, it is necessary to verify that the EHIS is correctly calculating charge (Z) for all ions, Hydrogen through Nickel, in all five energy bands. In order to begin the checkout of the alpha-kappa values for high energies, EHIS science data will be passively collected while the SU operates in its nominal operations mode. Pulse Height Analysis (PHA) data from EHIS telemetry stream will be analyzed on the ground and the alpha and kappa values that give the best fit to the elemental data will be determined. 


\subsubsection{MPS-Lo Voltage Bias Optimization}

The optimum operating voltage is known from ground testing; however, any time MPS-Lo is turned on after a long duration in storage the MCP voltages must be stepped up slowly in case there are species being desorbed from the MCPs themselves. Rapid turn-on of the voltage can lead to excessive noise, and potential arcing, which might damage the instrument.

To perform this test the high voltages (electron and ion) are turned on at a known, safe voltage level where the MCPs are on their threshold of operation resulting in valid electron/ion counts. From this point the voltage is stepped up one level at a time until the optimum voltage level is reached. For each step, science data is collected and analyzed to determine if the counts are as expected or if there is evidence of excessive noise or discharge. Once the MCP optimum voltage for on orbit operations has been established during the PLT phase, only instrument degradation over the life of the mission will cause this exercise to be repeated.

\subsubsection{Cross Calibration of the $+X$ SGPS and the $-X$ SGPS}

Ten days, not necessarily consecutive, of science data for both SGPS units will be collected in order to confirm the counts in the respective channels of the SGPS $+X$ and SGPS -X SUs are consistent with on-orbit predictions. Additionally, this data will be used to resolve any discrepancy between the two units due to initial calibration uncertainty. If necessary, the GPA coefficient tables will be updated to normalize the fluxes between the two SGPSs; e.g. counts for one SGPS may be $25 \%$ high and the other may be $25 \%$ low, causing a $50 \%$ discrepancy between the two units. Ultimately, the data from this test will establish the performance baseline for mission life trending.

\subsubsection{SGPS D3-D1 Logic Circuit Test}

Telescope 3 of the SGPS SU has the capability of employing an out-of-band rejection technique by enabling a logic circuit that generates a difference signal between Detector 3 and Detector 1 (D3-D1), thus effectively suppressing the rear entry particles. Based on ground calibration data and the effectiveness of the D3-D1 circuit, it is being recommended that this circuit be enabled for 2 of the energy channels in Telescope 3 for nominal operations and leave it disabled for one of the channels for nominal operations.

An on-orbit test will be performed to confirm the functionality and performance of the SGPS +X and SGPS -X D3-D1 logic circuits. Science data will be collected for the cases of D3-D1 circuits disabled, enabled and then disabled. The performance of the D3-D1 circuit depends on the energy distribution of high energy protons on orbit. The before and after measurements will enable an assessment of the energy distribution change and its effect on the D3-D1 performance.

\section{CONCLUSIONS}

The Space Weather instruments EXIS, SUVI, and SEISS on GOES-R are described in this paper and details of the ground calibrations and plans for on-orbit calibrations are presented. Brief descriptions of the series of tests designed for the PLT phase are provided. Scientists and engineers helped to design and distill these tests in order to provide the best understanding of the instrument behavior and the Space Weather knowledge they're expected to advance. Considering that these instruments enhance the knowledge of Space Weather community significantly while providing continuity with the earlier GOES missions (where overlap exists) and simultaneously providing new capabilities, we are confident that the GOES-R community is embarking on an exciting new phase.

\section{ACKNOWLEDGMENTS}

The authors would like to thank the GOES-R Flight Project, Dr. Frank Eparvier and Mr. Mike Anfinson of the Laboratory for Atmospheric and Space Physics at University of Colorado, Boulder, Mr. Dean Read and Mr. Chris 
Edwards of the Lockheed Martin Space Systems Company, and Dr. Gary Galica of the Assurance Technology

Corporation for the many years of technical interaction on the EXIS, SUVI, and SEISS, respectively.

\section{REFERENCES}

[1] Kalluri, S., Sullivan, P., and Goodman, S., "The next series of geostationary weather satellites," Meteorological Technology International, April 2015.

[2] Chamberlin, P.C., Woods, T.N., Eparvier, F.G., and Jones, A.R., "Next Generation X-Ray Sensor (XRS) for GOESR Satellite Series," Proc. of SPIE Vol. 7438 (2009).

[3] Eparvier, F.G., et al, "The extreme ultraviolet sensor (EUVS) for GOES-R," Solar Physics and Space Weather Instrumentation III, Proc. of SPIE Vol. 7438 (2009).

[4] Snow, M., McClintock, W.E., Crotser, D., and Eparvier, F.G., "EUVS-C: The Measurement of the Magnesium II Index for GOES-R EXIS," Proc. of SPIE Vol. 7438 (2009).

[5] Lemen, J.R., et al, "The Atmospheric Imaging Assembly (AIA) on the Solar Dynamics Observatory (SDO)," Solar Physics, (2012).

[6] Galica, G., Dichter, B.K., Golightly, M., Chi-Wa Tsui, C.-W, Lopate, C., Connell, J. J., "GOES-R Space Environment In-Situ Suite: instruments overview, calibration results, data processing algorithms, and expected onorbit performance," Proc. SPIE Vol. 9881 (2016).

[7] Martínez-Galarce, D., Soufli, R., Windt, D., Bruner, M., Gullikson, E., Khatri, S., Robinson, J., Baker, S., Prast, E., "Microroughness measurements and EUV calibration of the Solar Ultraviolet Imager multilayer coated mirrors," Proc. of SPIE Vol. 8501 (2012).

[8] Martínez-Galarce, D., Harvey, J., Bruner, M., Lemen, J.R., Gullikson, E., Soufli, R., Prast, E., Khatri, S., “A novel forward-model technique for estimating EUV imaging performance - design and analysis of the SUVI telescope, Proc. of SPIE Vol. 7732 (2010). 